ARTICLE

\title{
A porous proton-relaying metal-organic framework material that accelerates electrochemical hydrogen evolution
}

\author{
Idan Hod ${ }^{1,2}$, Pravas Deria1, Wojciech Bury ${ }^{1,3}$, Joseph E. Mondloch¹, Chung-Wei Kung ${ }^{1,2,4}$, Monica So ,
} Matthew D. Sampson ${ }^{5}$, Aaron W. Peters ${ }^{1,2}$, Cliff P. Kubiak ${ }^{5}$, Omar K. Farha, ${ }^{1,2,6}$ \& Joseph T. Hupp $p^{1,2,7}$

The availability of efficient hydrogen evolution reaction (HER) catalysts is of high importance for solar fuel technologies aimed at reducing future carbon emissions. Even though Pt electrodes are excellent HER electrocatalysts, commercialization of large-scale hydrogen production technology requires finding an equally efficient, low-cost, earth-abundant alternative. Here, high porosity, metal-organic framework (MOF) films have been used as scaffolds for the deposition of a Ni-S electrocatalyst. Compared with an MOF-free Ni-S, the resulting hybrid materials exhibit significantly enhanced performance for HER from aqueous acid, decreasing the kinetic overpotential by more than $200 \mathrm{mV}$ at a benchmark current density of $10 \mathrm{mAcm}^{-2}$. Although the initial aim was to improve electrocatalytic activity by greatly boosting the active area of the Ni-S catalyst, the performance enhancements instead were found to arise primarily from the ability of the proton-conductive MOF to favourably modify the immediate chemical environment of the sulfide-based catalyst.

\footnotetext{
${ }^{1}$ Department of Chemistry, Northwestern University, 2145 Sheridan Road, Evanston, Illinois 60208, USA. ${ }^{2}$ Argonne-Northwestern Solar Energy Research (ANSER) Center, Northwestern University, Evanston, Illinois 60208, USA. ${ }^{3}$ Department of Chemistry, Warsaw University of Technology, Noakowskiego 3, 00-664 Warsaw, Poland. ${ }^{4}$ Department of Chemical Engineering, National Taiwan University, Number 1, Sector 4, Roosevelt Road, Taipei 10617, Taiwan. ${ }^{5}$ Department of Chemistry and Biochemistry, University of California San Diego, 9500 Gilman Drive, La Jolla, California 92093-0358, USA. ${ }^{6}$ Faculty of Science, Department of Chemistry, King Abdulaziz University, Jeddah 21589, Saudi Arabia. ${ }^{7}$ Argonne National Laboratory, 9700 South Cass Avenue, Argonne, Illinois 60439, USA. Correspondence and requests for materials should be addressed to O.K.F. (email: o-farha@northwestern.edu) or to J.T.H. (email: j-hupp@northwestern.edu).
} 
S ubstantial effort is being invested in the design and development of efficient electrocatalytic hydrogen evolution technologies, as these hold promise for a future carbon-free energy economy ${ }^{1-4}$. In acid solutions, the hydrogen evolution reaction (HER) entails the electrochemical reduction of protons: $2 \mathrm{H}^{+}+2 \mathrm{e}^{-} \rightarrow \mathrm{H}_{2}$. Catalysts are needed to decrease the otherwise enormous kinetic overpotential required to drive the reaction at suitably high current densities, J. For solar energy applications, where the corresponding oxidation reaction is the four-electron conversion of water to $\mathrm{O}_{2}$, a commonly cited target $J$ value is $10 \mathrm{~mA} \mathrm{~cm}^{-2}$ (ref. 5). Provided that catalyst poisons are absent, selected noble metal electrodes (especially platinum electrodes) can support hydrogen evolution at high current densities under low kinetic overpotentials ${ }^{6}$. However, realization of large-scale hydrogen production requires the development of alternative low-cost electrocatalysts containing only highly abundant elements. A variety of candidate catalyst materials, including metal alloys ${ }^{7}$, carbides $^{8}$, phosphides ${ }^{9,10}$, borides $^{8}$ and nitrides ${ }^{11}$, have shown promising HER activity and stability in acidic solutions. In addition, several metal sulfides and selenides (MS/ $\mathrm{MS}_{2} / \mathrm{MSe} / \mathrm{MSe}_{2}$ where $\mathrm{M}=\mathrm{Co}, \mathrm{W}, \mathrm{Mo}, \mathrm{Fe}$ and $\left.\mathrm{Ni}\right)^{12-16}$ have likewise recently been observed to display promising catalytic behaviour for hydrogen evolution. Nevertheless, substantial improvements in catalytic performance are needed to match the kinetic superiority of platinum electrodes.

Complementary to changing the composition of the electrocatalyst, a useful strategy for obtaining a significant leap in performance is to employ a porous supporting scaffold and create a composite electrode ${ }^{17,18}$. (A third approach, illustrated by various enzymes and their artificial mimics, is to modify the environment of the catalytically active site so as to provide proximal $\mathrm{H}$-bond-donating or -accepting sites and/or protonrelay and -delivery channels $)^{19-21}$. Porous scaffolds can boost an electrocatalyst's performance, that is, decrease overpotentials, by: (a) boosting the areal density of catalytically active surface sites, (b) improving the electronic conductivity of the electrode and/or (c) facilitating proton delivery. In addition, it has been shown that for some materials, certain crystal faces are much more catalytically active than others; the most striking examples perhaps are edge versus plane sites of layered, two-dimensional molybdenum or tungsten dichalcogenides ${ }^{12,16,22}$. By decreasing the lateral dimensions of crystallites constituting these catalysts, both a greater fraction and a greater absolute number of highactivity sites can be exposed ${ }^{14}$. The limit of this approach (not explored here) would be the synthesis of materials in small-cluster form, where the majority of exterior atoms, rather than only a tiny fraction, possesses the appropriate chemical coordination and geometric arrangement for high catalytic activity.

We reasoned that porous, electrode-surface-immobilized, metal-organic framework (MOF) crystallites might be ideal templates for synthesis of high-areal-density versions of inorganic electrocatalysts. In particular, we hypothesized that electrodeposition of nickel sulfide within the channels of the supported MOF might yield the desired composite or hybrid, nanostructured catalysts. Although underexplored as an HER electrocatalyst, especially in acidic environments, the electrodeposition of nickel sulfide is reasonably well understood ${ }^{23,24}$. Furthermore, a variety of sulfides of other first-row transition metals have been shown to be electrocatalytically competent, at modest kinetic overpotentials, for hydrogen evolution from water at low $\mathrm{pHs}^{12-16}$. As a candidate template or scaffold, we chose NU1000 (ref. 25), a mesoporous MOF that can be grown solvothermally on conductive glass as a film comprising rodshaped crystallites of a few microns length and a fraction of a micron width ${ }^{26}$. NU-1000 is one member of a growing family of porous MOFs featuring hexa-zirconium oxo/hydroxo/aquo clusters as nodes ${ }^{27-31}$ and displaying exceptional resistance to hydrolysis ${ }^{32-34}$, even at elevated temperature and/or in the presence of hydronium or hydroxide ions. Thus, this material was anticipated to be sufficiently chemically stable to function as a component of a hybrid HER electrocatalyst. It should be noted that various MOFs that anchor or encapsulate noble-metal nanoparticles or biomimetic molecular catalysts have recently been used in combination with molecular chromophores and sacrificial reductants (hole scavengers) to demonstrate photochemical hydrogen evolution ${ }^{35-40}$.

Here we report on the electrochemical assembly of a surface immobilized hybrid of Ni-S (we intentionally use Ni-S (rather than NiS) to denote a material comprising nickel and sulfur, but of initially unspecified metal:non-metal stoichiometry; we subsequently specify more precisely the composition of the form of Ni-S electrodeposited here) and NU-1000, and on the performance of the hybrid as an HER electrocatalyst in water at $\mathrm{pH}$ 1. The hybrid assembly yields a benchmark cathodic current of $10 \mathrm{~mA} \mathrm{~cm}^{-2}$ (together with $\mathrm{H}_{2}$ bubbles) at iR-corrected overpotentials ( $\eta$-values) as low as $238 \mathrm{mV}$. Under the same conditions, and at the same benchmark current density, the observed overpotential with flat, MOF-free Ni-S films as electrocatalysts is $443 \mathrm{mV}$.

\section{Results}

Electrocatalyst synthesis. Illustrations of the crystal structure of NU-1000, its $\mathrm{Zr}_{6}\left(\mu_{3}-\mathrm{O}\right)_{4}\left(\mu_{3}-\mathrm{OH}\right)_{4}(\mathrm{OH})_{4}\left(\mathrm{OH}_{2}\right)_{4}$ nodes $^{41}$ and its organic linkers are presented in Fig. 1. The crystal structure shows the existence of both triangular and hexagonal one-dimensional channels; together with smaller apertures (ca. 8 A diameter) along the channel walls, they are responsible for the high porosity of the material (the diameters of the one-dimensional channels are $c a$. 10 and $31 \AA$. In addition, around $20 \%$ of the hexagonal pores contain an extra node at the hexagon centre. The extra node is connected to the rest of the MOF via additional TBAPy ${ }^{4-}$ linkers. For simplicity, these are omitted from Fig. 1. $\mathrm{N}_{2}$ adsorption measurements (bulk samples) show that the MOF void volume is $c a .71 \%$ of the total volume). Figure 2 a shows a scanning electron microscopy (SEM) image of a NU-1000_Ni-S electrode ( 2 min of Ni-S deposition, see also Supplementary Fig. 1 for experimental conditions), featuring the hexagonal rod-shaped crystals that are typical for NU-1000, whereas Fig. 2b shows a cross-sectional SEM view of a NU-1000_Ni-S electrode. X-ray Photoelectron Spectroscopy (XPS) measurements of the NU1000_Ni-S film (Supplementary Fig. 2) confirm the presence of both nickel and sulfur. The observed $S 2 p$ binding energy is $c a$. $162 \mathrm{eV}$, implying sulfur incorporation as $\mathrm{S}^{2-}$. The presence of a peak at $168 \mathrm{eV}$ is indicative of sulfur in a higher oxidation state, presumably oxy-sulfur species that might well arise from surface oxidation (air oxidation) of $\mathrm{Ni}-\mathrm{S}^{15}$. The $\mathrm{Ni} 2 p$ region shows a broad peak at a binding energy of centred around $860 \mathrm{eV}$, which can be attributed to $\mathrm{Ni}^{2}+$ as well as to other nickel oxidation states $^{42}$.

Elemental analysis of the hybrid films using both energy dispersive X-ray spectroscopy (EDS) and inductively coupled plasma-optical emission spectroscopy (ICP-OES), corroborate and quantify the conclusion that the hybrid films contain $\mathrm{Ni}$ and S. The EDS-obtained ratio of Ni:S was 1.7:1.0. ICP-OES yields a similar $\mathrm{Ni}$ S ratio of $1.6: 1.0$, suggesting $\mathrm{Ni}_{3} \mathrm{~S}_{2}$ as a primary deposition product (see Supplementary Table 1). EDS crosssectional mapping of an MOF rod revealed, however, that only trace amount of nickel and sulfur are present in the extended portion of the MOF rods (Supplementary Fig. 3). Instead, these elements are confined and concentrated as a planar film, as illustrated in the bottom section of Fig. 3. As the MOFs 


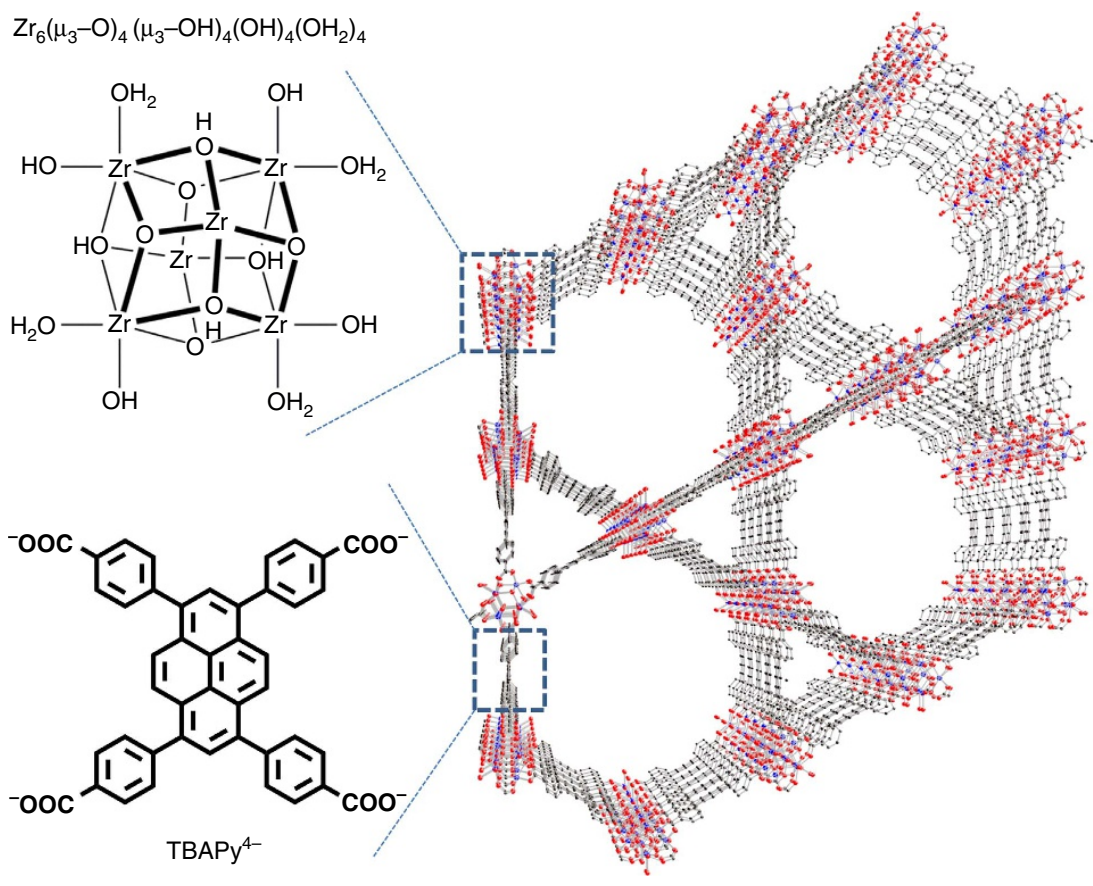

Figure 1 | Schematic representation. Schematic representation of NU-1000's crystal structure.
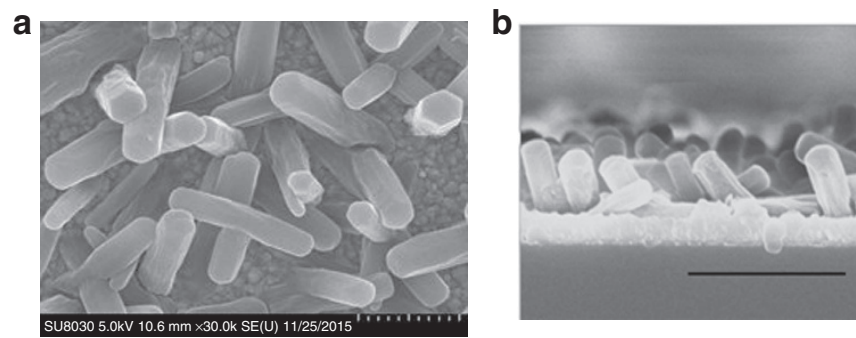

Figure 2 | SEM images of NU-1000_Ni-S films. (a) SEM image of an NU-1000_Ni-S film showing the typical hexagonal rod-shaped crystals of NU-1000 on top of the FTO substrate (scale bar, $1 \mu \mathrm{m}$ ). (b) Cross-sectional SEM image of NU-1000_Ni-S film (scale bar, $2 \mu \mathrm{m}$ ).

themeslves are insulating in the region of potential where catalytic evolution of $\mathrm{H}_{2}$ occurs, the MOF-distributed traces of nickel and sulfur are electrochemically inaccessible and, therefore, unable to participate in electrocatalysis (HER electrocatalysis by metal sulfides generally requires proximal pairs of sulfur atoms (ions). The loading of sulfur in the extended region of the MOF, however, is $<0.5$ sulfur ions per hexa-zirconium node. Thus, even if the MOF could be rendered conductive, it is unlikely that these sulfur atoms would be catalytic).

Powder X-ray diffraction (XRD) measurements of putative FTO_NU-1000 films confirm that the observed crystallites consist of NU-1000 (Supplementary Fig. 4). Notably, the MOF's crystal structure remains intact. However, no peaks attributable to nickel sulfide are observed, suggesting that the material deposited is amorphous, in agreement with previous reports ${ }^{23}$.

More detailed information about the composition of the electrodeposited material was obtained via Raman spectroscopy. As can be seen in Supplementary Fig. 5, the Raman spectrum of a Ni-S MOF hybrid film contains the signature vibrational peaks of $\mathrm{Ni}_{3} \mathrm{~S}_{2}\left(176,190,217,300,319 \text { and } 341 \mathrm{~cm}^{-1}\right)^{43}$. In addition, it is important to note that small peaks are present, corresponding to

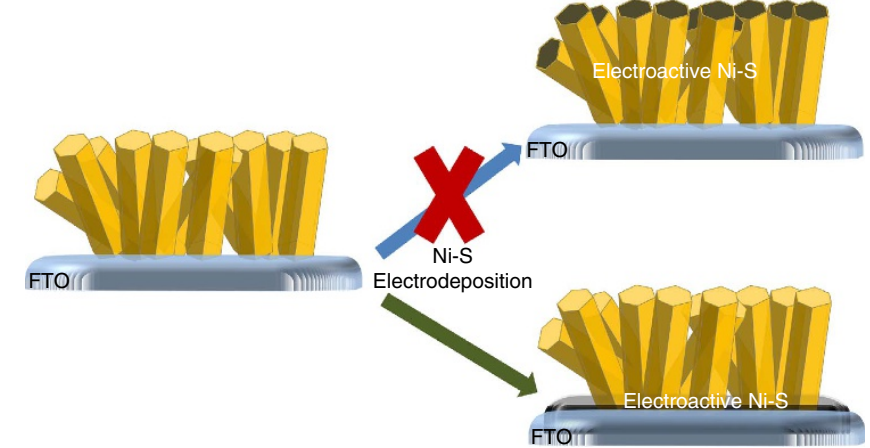

Figure 3 | Illustration of Ni-S electrodeposition to create the NU1000_Ni-S hybrid system. The initial aim was to use the MOF as a template for depositing high surface area Ni-S rods (top section). However, $\mathrm{Ni}-\mathrm{S}$ was deposited as a flat layer (bottom section).

$\mathrm{NiS}_{2}$ (ref. 44) (276, 447 and $\left.465 \mathrm{~cm}^{-1}\right)$ and $\mathrm{NiS}\left(379 \mathrm{~cm}^{-1}\right)^{45}$. The Raman spectroscopy results combined with the EDS and ICP-OES elemental analysis establish that the main electrodeposition product is $\mathrm{Ni}_{3} \mathrm{~S}_{2}$.

HER catalysis. As suggested by the top portion of Fig. 3, our initial aim was simply to boost the metal-sulfide electrode surface area by using the MOF as a porous template for the electrodeposition of mesoscale nickel sulfide rods. In contrast to our expectations, however, the MOF did not function as a physical template and, consequently, did not increase the electrode surface area. Instead, Ni-S deposited as a flat film that in-filled the bottoms of the MOF rods, while coating both the bare conductive glass platform (fluorine-doped tin oxide, FTO) and the portions of FTO residing at the bottom of each MOF channel (see Fig. 3).

Notably, these channels are wide enough to readily allow permeation by the electrolyte solution in a hydrogen-evolution 
cell. As such, the MOF channels help to define the local chemical environment of the portion of the deposited $\mathrm{Ni}-\mathrm{S}$ that is in contact with the electrolyte solution. As will be discussed further below, it is this local environment that alters the catalytic activity of the metal-sulfide electrode and yields the observed substantial decrease in HER overpotential.

To assess the electrocatalytic properties of the hybrid assembly towards hydrogen evolution, we studied four types of electrodes: bare FTO, a MOF scaffold grown on FTO substrate (FTO_NU1000), Ni-S deposited on FTO substrate (FTO_Ni-S) and Ni-S deposited on FTO-supported NU-1000 (NU-1000_Ni-S). J-V curves and Tafel plots ( $V$ versus $\log J$ ) of the different systems in aqueous $0.1 \mathrm{M} \mathrm{HCl}(\mathrm{pH} 1)$ are shown in Fig. 4a. As expected, the bare FTO electrode exhibits poor electrocatalytic activity towards the HER, with a high reaction onset potential of around $600 \mathrm{mV}$ versus Reversible Hydrogen Electrode (RHE) and an overpotential of $c a .1 \mathrm{~V}$ at $J=10 \mathrm{~mA} \mathrm{~cm}^{-2}$. For Ni-S directly electrodeposited on FTO (only), the overpotential at $10 \mathrm{~mA} \mathrm{~cm}^{-2}$ is $c a$. $560 \mathrm{mV}$ lower, clearly illustrating its ability to function as an electrocatalyst. Addition of the MOF scaffold, that is, formation of the hybrid assembly, further decreases the overpotential $10 \mathrm{~mA} \mathrm{~cm}^{-2}$ to $238 \mathrm{mV}$ (this was the lowest iR-corrected overpotential observed based on several electrodes. The values occasionally ranged as a high as $280 \mathrm{mV}$ ). Finally, a control experiment with FTO_NU-1000, but no metal sulfide, yields a kinetic overpotential at $10 \mathrm{~mA} \mathrm{~cm}^{-2}$ of $c a .640 \mathrm{mV}$. Although the value in isolation is unremarkable, the $c a .360 \mathrm{mV}$ decrease in $\eta$ relative to the MOF-free bare FTO electrode is both substantial and unexpected. The isolated MOF contains no components capable of redox mediation and there are no obvious sites for adsorption and stabilization of potential reaction intermediates such as neutral hydrogen atoms.

Returning to the hybrid Ni-S catalyst, the observed kinetic overpotential at $10 \mathrm{~mA} \mathrm{~cm}^{-2}$ compares reasonably well with recently reported results for several other non-noble metal electrocatalysts for the HER in aqueous acid. For example, an $\mathrm{NiS}_{2}$ electrocatalyst showed only $4 \mathrm{mAcm}^{-2}$ at around $240 \mathrm{mV}$ of overpotential, $\mathrm{Ni}_{3} \mathrm{~S}_{2}$ exhibit $10 \mathrm{~mA} \mathrm{~cm}^{-2}$ at $213 \mathrm{mV}$ of overpotential, whereas $\mathrm{CoS}_{2}$ and $\mathrm{FeS}_{2}$ yielded $10 \mathrm{~mA} \mathrm{~cm}^{-2}$ at $\eta$-values of ca. 230 and $260 \mathrm{mV}$, respectively (all in $0.5 \mathrm{M} \mathrm{H}_{2} \mathrm{SO}_{4}$, $\mathrm{pH} \mathrm{0}$; it is noteworthy that $\mathrm{pH} 1$ was used in this work ${ }^{13,24}$. In neutral (pH 7) solutions, $\eta$-values of $<160 \mathrm{mV}$, at $10 \mathrm{~mA} \mathrm{~cm}^{-2}$, have been reported for electrodeposited and subsequently annealed $\operatorname{CoS}^{46}$.

The Ni-S mass loading on NU-1000_Ni-S films during electrodeposition was measured in situ using electrochemical quartz crystal microbalance (EQCM) techniques (see Methods section). As can be seen in Supplementary Fig. 6a, after 2 min of electrodeposition, Ni-S loading in NU-1000_Ni-S is $28 \mu \mathrm{g} \mathrm{cm}^{-2}$ $\left(0.116 \mu \mathrm{mol} \mathrm{cm}^{-2}\right)$. Potentiostatic electrolysis of NU-1000_Ni-S for a period of over $2 \mathrm{~h}$ at overpotential of $210 \mathrm{mV}$ was done, while recording the charge flowing through the system (Supplementary Fig. 6b). From this charge, we have been able to estimate the amount of $\mathrm{H}_{2}$ formed during the electrolysis (taking into account the $\mathrm{H}_{2}$ Faradic yield of $93 \%$, as determined by gas chromatography based on electrolysis at $2 \mathrm{~mA} \mathrm{~cm}^{-2}$ for $3 \mathrm{~h}$; see Supplementary Fig. 7). We note that following previous reports, we suggest that Faradaic efficiency falls short of $100 \%$ due to $\mathrm{H}_{2}$ bubble formation on the surface of the electrode ${ }^{15,47}$. If we assume that only the portion of the metal sulfide that is in contact with the solution is catalytic (that is, the electrolyte does not permeate and swell the metal sulfide), and if we assume that an adjacent pair of nickel-coordinated sulfide ion constitutes one HER catalytic site, we obtain an active site normalized (upper limit) turnover frequency (TOF) of $34 \mathrm{~s}^{-1}$ and a turnover number (TON) of 273,000 (based on $8,000 \mathrm{~s}$ of electrolysis at a constant potential of $-210 \mathrm{mV}$ versus RHE; see Methods section for details). For the total amount of Ni-S in the NU-1000_Ni-S film $\left(0.116 \mu \mathrm{mol} \mathrm{cm}^{-2}\right)$, TOF and TON values were calculated to be $0.208 \mathrm{~s}^{-1}$ and 1668 , respectively.

As a preliminary test of the stability of the NU-1000_Ni-S combination, galvanostatic electrolysis measurements were conducted at $10 \mathrm{~mA} \mathrm{~cm}^{-2}$, in aqueous $\mathrm{HCl}$ at $\mathrm{pH} 1$ (Fig. 5a). Notably, the overpotential needed to produce the imposed current remains constant $( \pm 20 \mathrm{mV})$ for $2 \mathrm{~h}$ of measurement, indicating that the NU-1000_Ni-S system can sustain its catalytic activity for relatively long periods of time. Power XRD measurements taken on a hybrid-catalyst film before and after $2 \mathrm{~h}$ of galvanostatic electrolysis at $10 \mathrm{~mA} \mathrm{~cm}^{-2}$ show that the MOF scaffold retains its crystallinity under electrocatalysis working conditions (Fig. 5b). To further assess the stability of the hybrid film during catalysis, we recorded a ultraviolet-visible spectrum of the $\mathrm{pH} 1$ electrolyte solution after $2 \mathrm{~h}$ of galvanostatic electrolysis at $10 \mathrm{~mA} \mathrm{~cm}^{-2}$ (Supplementary Fig. 8), monitoring the wavelengths at which 1,3,6,8-tetrakis(p-benzoic acid)pyrene $\left(\mathrm{H}_{4}\right.$ TBAPy $)$ linkers exhibit maximum absorbance $(\sim 390 \mathrm{~nm})$. The electrolysis solution displays no traces of leached NU-1000 linker, indicating that the MOF remains intact over the course of $2 \mathrm{~h}$ of catalysis. (For comparison, a spectrum of a digested NU-1000_Ni-S film is presented, showing a peak at $390 \mathrm{~nm}$ attributable to the linker.)

Origin(s) of enhanced catalytic performance by hybrid assemblies. It is well known that in certain cases Tafel slopes can serve as indicators of the rate-determining step in the HER, as well as

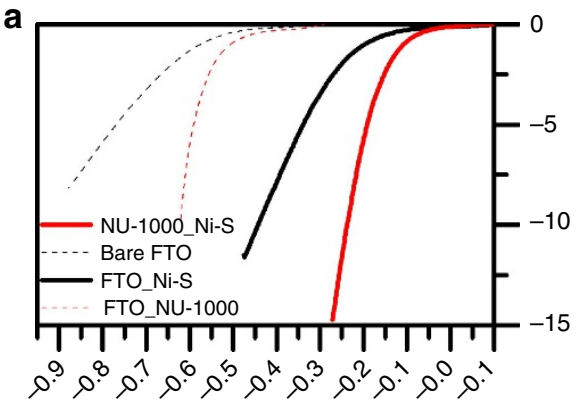

Potential ( $V$ vs RHE)

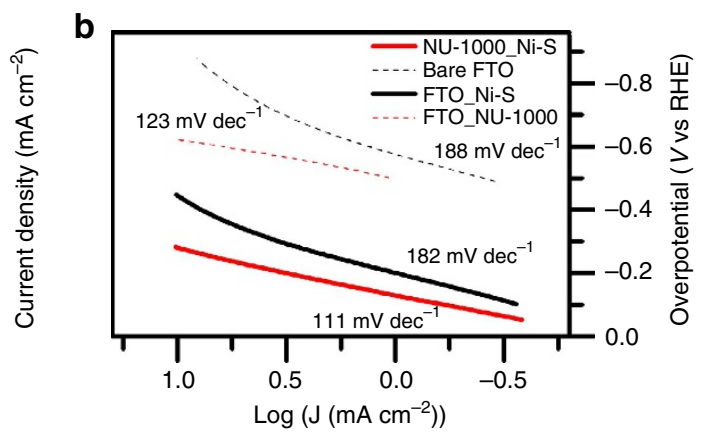

Figure 4 | Comparison between the electrocatalytic HER performance. Four types of electrodes: bare FTO (dotted black), FTO_NU-1000 (dotted red), FTO_Ni-S (black) and NU-1000_Ni-S (red). (a) J-V curves. (b) Tafel plots. The listed values of 182 and $188 \mathrm{mV} \mathrm{dec}^{-1}$ for the HER Tafel slopes of the bare FTO and FTO_Ni-S samples refer to the low current density region only. The slopes clearly are larger at higher current densities. 
indicate the existence of other kinetically relevant effects ${ }^{48}$. Figure $4 \mathrm{~b}$ shows Tafel plots ( $V$ versus log $J$ plots) for the four types of electrodes examined here. For the MOF-free electrodes, with or without Ni-S, the slopes of the plots are near $180 \mathrm{mV}$ per decade of current density, whereas for the NU-1000-functionalized electrodes the slopes are near $120 \mathrm{mV} \mathrm{dec}^{-1}$. Values substantially $>120 \mathrm{mV}$ typically are indicative of the presence of an uncompensated resistive element-for example, the resistance of the catalyst itself ${ }^{49}$.

If we assume that in the presence of the MOF scaffold, complicating factors are absent and the Tafel slope reflects only interfacial kinetics, the observed values of $c a$. $120 \mathrm{mV} \mathrm{dec}^{-1}$ imply that the HER is governed by either the Volmer-Heyrovsky or Volmer-Tafel mechanism (see Supplementary Note 1 for HER mechanistic details). Both mechanisms involve rate-limiting reduction of a proton to yield a catalyst-adsorbed $\mathrm{H}$ atom. For the Volmer-Heyrovsky mechanism, this step is followed by fast electrochemical reduction of a second proton and concomitant formation of an $\mathrm{H}-\mathrm{H}$ bond. For the Volmer-Tafel pathway, the initial step is followed by fast formation of an $\mathrm{H}-\mathrm{H}$ bond between a pair of surface-bound hydrogen atoms to form $\mathrm{H}_{2}$. It is noteworthy that the latter mechanism requires the catalyst to offer an immediately proximal pair of $\mathrm{H}$-atom adsorption sitesfor example, a pair of surface-exposed sulfide ions.

Still to be answered is why MOF-Ni-S hybrid catalyst formation decreases the overpotential for Ni-S-catalysed $\mathrm{H}_{2}$ evolution at $10 \mathrm{~mA} \mathrm{~cm}^{-2}$ by more than $200 \mathrm{mV}$. Our initial hypothesis, dispelled by SEM and EDS results mentioned above, was that catalyst electrodeposition within the MOF scaffold would greatly boost its surface area. Nevertheless, the presence of the MOF might still serve to roughen the electrodeposited film and therby increase its effective surface area. Cyclic voltammetry (CV) measurements of electrochemical currents in the voltage range positive of the hydrogen evolution region, that is, capacitive currents associated with electrical double-layer charging, are expected to scale as the solution-accessible surface area of the electrocatalys $5^{50}$. Comparison of these currents over a range of voltammetric sweep rates (Fig. 6c) indicates remarkably similar surface areas. Indeed, the electroactive surface area for the hybrid assembly is only about 1.5 times greater than that for the simple $\mathrm{Ni}-\mathrm{S}$ film, that is, far too little to account for the change in overpotential.

If the number of electrocatalytically active sites is little changed by introduction of the MOF scaffold, we are left with alterations in the local reaction environment as the most probable source of the observed reactivity enhancement. The putative local environment effects could conceivably take the form of electronic modulation of the properties of Ni-S, perhaps involving the creation of highly catalytic sites at MOF-engendered grain boundaries. Such effects could be especially important for twodimensional layered compounds such as $\mathrm{MoS}_{2}$, but seem less likely to enormously influence the activity of an amorphous, three-dimensional material such as $\mathrm{Ni}$-S.

To gain insight into the possible importance of electronic effects on the hybrid system HER performance, we employed electrochemical impedance spectroscopy with FTO_Ni-S and NU-1000_Ni-S electrodes, taken under HER working conditions. Nyquist plots show a resistance element which is attributed to solution voltage drops, in series with one semicircle
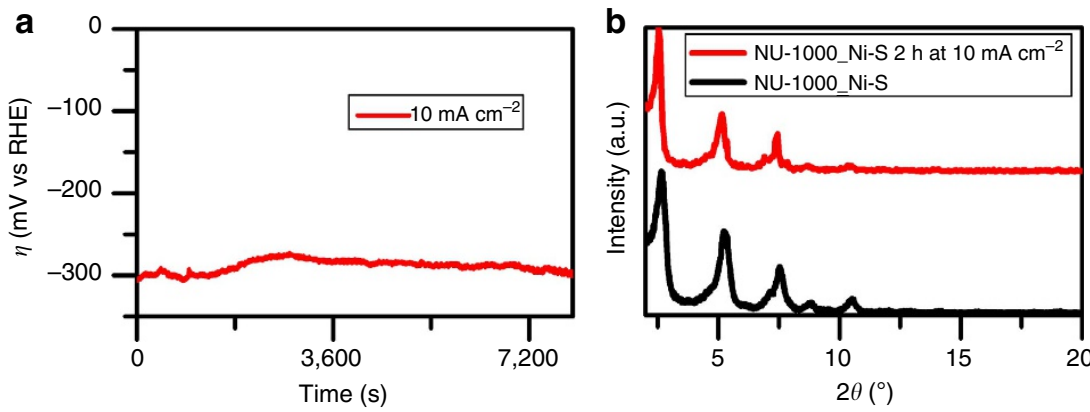

Figure 5 | Stability analysis of NU-1000_Ni-S under HER working conditions. (a) Galvanostatic electrolysis measurement of NU-1000_Ni-S system under a constant current of $10 \mathrm{~mA} \mathrm{~cm}^{-2}$, showing the voltage stability of the system for more than $2 \mathrm{~h}$. (b) Powder XRD plots for an NU-1000_Ni-S film before and after galvanostatic electrolysis measurement under a constant current of $10 \mathrm{~mA} \mathrm{~cm}^{-2}$ for $2 \mathrm{~h}$. The plots show that framework crystallinity is retained.
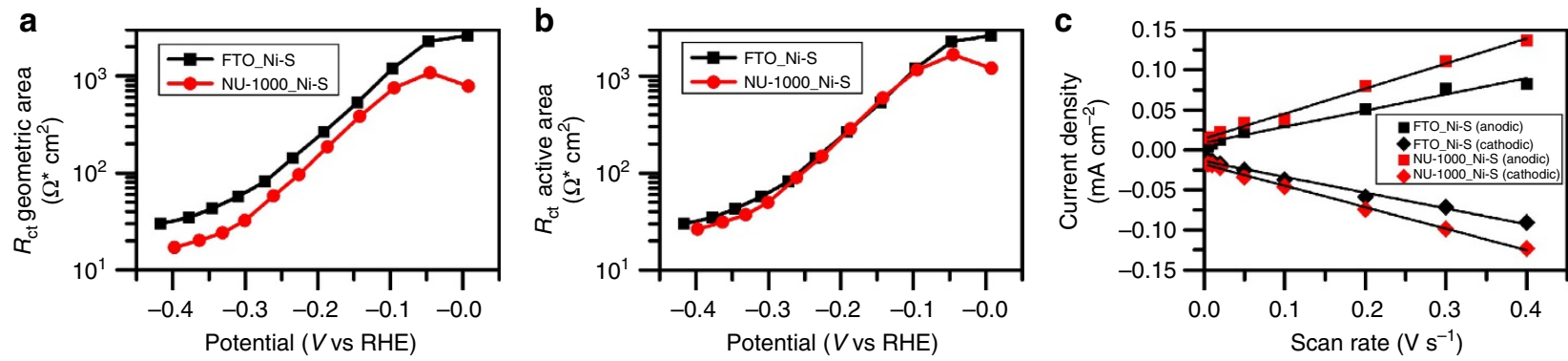

Figure 6 | EIS analysis of the MOF's scaffold impact on the electronic properties of Ni-S. (a) A plot of geometric area normalized charge transfer resistance $\left(R_{\mathrm{ct}}\right)$ versus applied potential, comparing between FTO_NiS and NU-1000_Ni-S electrodes. (b) A plot of electroactive surface area normalized $R_{\mathrm{ct}}$ versus applied potential, exhibiting similar $R_{\mathrm{ct}}$ values for both FTO_Ni-S and NU-1000_Ni-S electrodes. (c) A plot of double-layer capacitive current versus CV scan rate. The slope of each curve is relative to the amount of electroactive surface area of the system. 
a

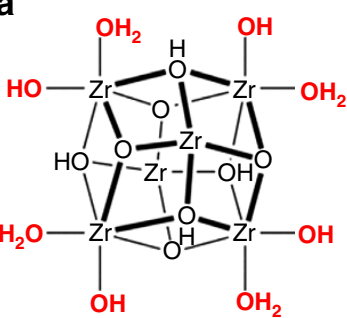

b

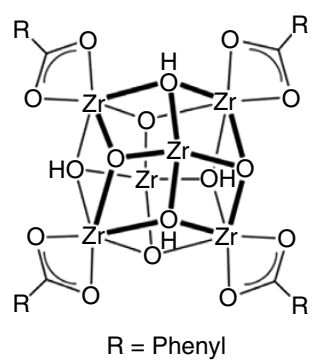

Figure 7 | Effect of $\mathrm{Zr}_{6}$ based node's terminal $-\mathrm{OH}$ and $-\mathrm{OH}_{\mathbf{2}}$ ligands on HER activity. (a) NU-1000's $\mathrm{Zr}_{6}$ based node, containing the terminal $-\mathrm{OH}$ and $-\mathrm{OH}_{2}$ ligands (marked in red). (b) Schematic representation of a benzoate-modified NU-1000 $\mathrm{Zr}_{6}$ based node. The benzoate replaces the node's terminal $-\mathrm{OH}$ and $-\mathrm{OH}_{2}$ ligands. Omitted, for simplicity, are atoms associated with coordinated linkers.

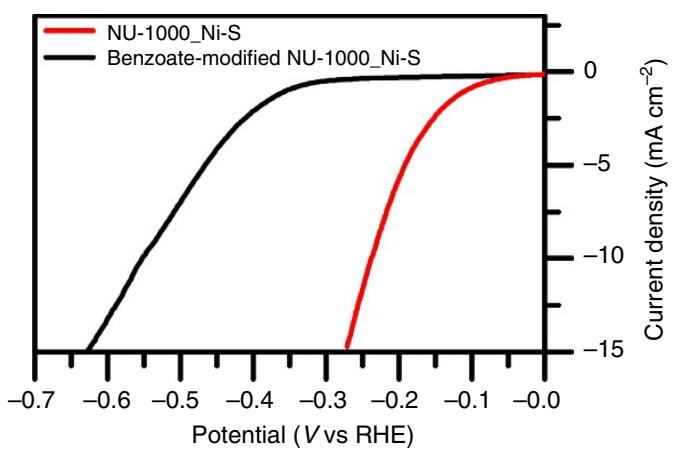

Figure 8 | Effect of $\mathbf{Z r}_{6}$ based node's terminal $-\mathrm{OH}$ and $-\mathrm{OH}_{\mathbf{2}}$ ligands, $\mathbf{J}-\boldsymbol{V}$ curves. Comparison between the catalytic performance of NU-1000_Ni-S (red) and benzoate-modified NU-1000_Ni-S (black). Substitution of the terminal $-\mathrm{OH}$ and $-\mathrm{OH}_{2}$ ligands by benzoate ligands significantly reduces the HER catalytic activity of the hybrid film.

corresponding to the parallel contribution of both catalyst's chemical capacitance and charge transfer resistance $\left(R_{\mathrm{ct}}\right)$ at the catalyst/solution interface (see Supplementary Fig. 9$)^{15}$. Figure 6 presents plots of $\log R_{\mathrm{ct}}$ versus $V$ for the two types of electrodes without (Fig. 6a) and with (Fig. 6b) correction for the minor difference in catalyst surface area. The plots show in striking manner that the interfacial electron-transfer step, in isolation, is not influenced by the presence of the MOF scaffold.

We next considered the possibility that catalytically important modification of the active-site environment could be assosiated with the solution side of the interface. The aquo- and hydroxorich nodes of the MOF (see Fig. 7a) could, for example, alter the local proton activity, as distinct from the bulk solution activity, or the MOF's nodes might facilitate local proton delivery and/or long-range proton transport.

Although we lack specific insight into about how the aquo- and hydroxo-functionalized MOF node might assist Ni-S in catalysing the HER, we reasoned that largely eliminating these ligands could provide an indication of their importance. We assembled a variant of NU-1000_Ni-S using 'as-synthesized' NU-1000 in which benzoate ligands replace the node's terminal $-\mathrm{OH}$ and $\mathrm{OH}_{2}$ ligands ${ }^{25,41,51,52}$ (see Fig. $7 \mathrm{~b}$ for $\mathrm{Zr}_{6}$-based node structure illustration and Supplementary Fig. 10 for ${ }^{1} \mathrm{H}$-NMR characterization of benzoate-modified NU-1000, showing the incorporation of four benzoates per $\mathrm{Zr}_{6}$ node). As shown by the $J-V$ comparisons in Fig. 8, benzoate substitution eliminates the co-catalytic behaviour of the MOF scaffold, now requiring an
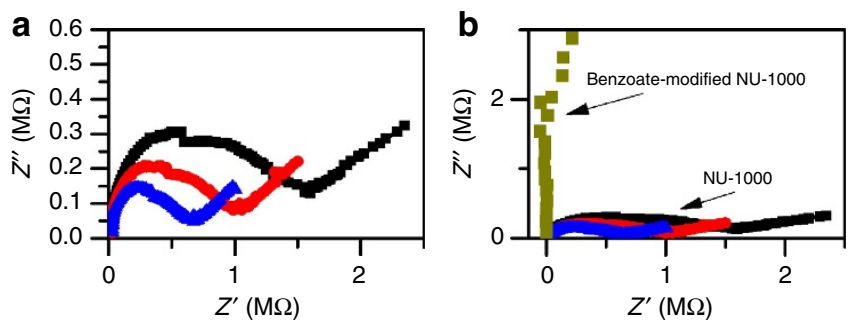

Figure 9 | Proton conductivity measuremets. Nyquist plots of $A C$ impedance measurements of proton conductivity of (a) NU-1000 in contact with humid air. As exposure time increases, the arc size decreases. (b) Comparison between Nyquist plots of NU-1000 and benzoate-modified $\mathrm{NU}-1000$. The observed large resistance for the benzoate-modified sample is equivalent to low conductivity.

overpotential of $553 \mathrm{mV}$ at $10 \mathrm{~mA} \mathrm{~cm}^{-2}$. SEM images (top view and cross-section) of the benzoate-modified NU-1000 show that the MOF's crystal morphology has not changed and the film exhibits similar inter-rod spacing for the electrodeposition of Ni-S (Supplementary Fig. 11a). In addition, EQCM data shown on Supplementary Fig. 11b reveal similar mass loadings of Ni-S in the benzoate-modified NU-1000 film $\left(26.7 \mu \mathrm{g} \mathrm{cm}^{-2}\right)$ compared with aquo- and hydroxo-functionalized NU-1000 $\left(28 \mu \mathrm{g} \mathrm{cm}^{-2}\right)$. The results in Fig. 8 clearly demonstrate the importance of the node's terminal $-\mathrm{OH}$ and $-\mathrm{OH}_{2}$ ligands to the HER performance of the hybrid system. As one would anticipate, re-installing benzoate ligands on the MOF nodes and thereby displacing nodecoordinated aqua and hydroxo ligands ${ }^{51-53}$ reverse the catalytic enhancement and yield HER $J-V$ behaviour similar to that of the benzoate-containing 'as-synthesized' version of the hybrid metalsulfide/MOF system; see Supplementary Fig. 12.

To gauge whether the aquo-ligated version of the MOF scaffold has the potential to assist proton transport, we examined its proton conductivity via impedance spectroscopy during and after infiltration with water from a high humidity atmosphere. A substantial literature on proton conductivity in MOFs now exists $^{54-57}$. One means of engendering conductivity in MOFs is by the presentation of Bronsted acids or bases to an infiltrating hydroxylic solvent. Although $\mathrm{p} K_{\mathrm{a}}$ and $\mathrm{p} K_{\mathrm{b}}$ values have yet to be determined for the poly-protic nodes of NU-1000, clearly there is the potential for acid/base reactivity. Figure 9a shows representative Nyquist plots for a measurement of a pellet of NU-1000. The plots are characterized by a single arc, attributable to proton conductivity. The diameter of the arc decreases with time, reflecting progressive uptake of water and indicating increasing conductivity. The conductivity obtained at equilibrium is $2 \times 10^{-7} \mathrm{~S} \mathrm{~cm}^{-1}$. Although of only modest magnitude, the observed conductivity implies the ability of the scaffold to assist in proton delivery to proximal catalytic sites. The extent to which such assistance is of value in aqueous $0.1 \mathrm{M}$ $\mathrm{HCl}$ remains to be determined. In comparison with NU-1000, Nyquist plots for a measurement of a pellet of benzoate-modified NU-1000 (Fig. 9b) revealed such a significant increase in the arc's diameter (larger resistance or smaller conductivity) that no meaninful data fitting could be obtained. These measurements confirm that the node's terminal $-\mathrm{OH}$ and/or $\mathrm{OH}_{2}$ ligands are essential for obtaining measurable proton conductivity.

\section{Discussion}

In conclusion, hybrid electrocatalysts consisting of electrodeposited Ni-S and solvothermally grown, electrode-supported NU-1000, a mesoporous, acid-stable MOF, display very good activity for hydrogen evolution. In aqueous $\mathrm{HCl}$ at $\mathrm{pH} 1$, the 
hybrid material can deliver a catalytic current of $10 \mathrm{~mA} \mathrm{~cm}^{-2}$ at an iR-corrected overpotential of just $238 \mathrm{mV}-\mathrm{a}$ sizable (ca. $200 \mathrm{mV}$ ) decrease in overpotential relative to MOF-free Ni$\mathrm{S}$ films as electrocatalysts. The hybrid probably catalyses the reaction via the Volmer-Tafel mechanism, as evidenced in part by Tafel slopes near $120 \mathrm{mV} \mathrm{dec}^{-1}$ for plots of $\log J$ versus overpotential. Despite its substantial porosity and high surface area, the MOF scaffold does not exert its co-catalytic effect by boosting the electroactive surface area of the subsequently deposited nickel sulfide. Instead, it serves to modify the immediate environment of the electrocatalyst, rendering it more favourable for local proton delivery and/or transport. Thus, the aquo- and hydroxo-rich nodes of the MOF appear to contribute in a manner that is reminiscent of organic acids or bases in proximity to metal-ion-based cofactors in enzymes that catalyse proton-coupled electron-transfer reactions, a concept that has likewise been exploited in the design of abiotic molecular catalysts for both hydrogen ${ }^{58-61}$ and oxygen ${ }^{62}$ evolution, as well as $\mathrm{CO}_{2}$ reduction $^{63}$.

\section{Methods}

Chemicals. All chemicals, benzoic acid (Aldrich, 99.5\%), zirconyl chloride octahydrate (Aldrich, 98\%), N,N-dimethylformamide (DMF) (Macron, 99.8\%), hydrochloric acid ( $\mathrm{HCl}, 36.5 \%-38.0 \%$, Macron), acetone (Macron), $\mathrm{NiCl}_{2}$ (Aldrich, $98 \%$ ) and thiourea (Sigma, 99\%) were used as received without further purification. Deionized water was used throughout the work. The chemicals used for the synthesis of the $\mathrm{H}_{4}$ TBAPy linkers were the same as those reported in our previous work $^{25}$.

Instrumentation. Thin-film XRD patterns were measured on a Rigaku ATX-G thin-film diffraction workstation.

SEM images and EDS mapping were measured on a Hitachi SU8030 SEM.

For ICP-OES experiments, two samples of the NU-1000_Ni-S thin film were scraped from their substrates and collected into a microwave vial $(4 \mathrm{ml})$. Then, $0.25 \mathrm{ml}$ concentrated $\mathrm{H}_{2} \mathrm{O}_{2}$ and $0.75 \mathrm{ml}$ concentrated $\mathrm{H}_{2} \mathrm{SO}_{4}$ were added. The vial was capped and irradiated in a microwave oven at $150{ }^{\circ} \mathrm{C}$ for $5 \mathrm{~min}$. The resultant clear solution was diluted to $25 \mathrm{ml}$ with nanopure water and analysed via ICP-OES (Varian Vista MPX instrument). Ni, S and Zr concentration were calculated from external stock solutions.

Raman spectroscopy measurements were made using an Acton TriVista Confocal Raman Spectroscopy System. Sample irradiation was done with a 514.5-nm laser. The acquisition time was $60 \mathrm{~s}$ and the reported spectrum was obtained by averaging ten runs.

All CV and impedance spectroscopy experiments were performed on a Solarton Analytical Modulab Potentiostat equipped with an FRA Impedance module. A three-electrode electrochemical setup was used, with a platinum mesh counter electrode and $\mathrm{Ag} / \mathrm{AgCl} / \mathrm{KCl}$ (sat'd) electrode as reference electrode. Electrochemical data were measured in aqueous $0.1 \mathrm{M} \mathrm{HCl}(\mathrm{pH} 1)$ solutions and were adjusted to RHE scale by adding $(0.197+0.059 \times \mathrm{pH}) \mathrm{V}$ to the measured potential. iR corrections were made to the obtained $i-V$ curves according to the series resistances measured on the same electrochemical setup using impedance spectroscopy.

EQCM experiments were conducted with a MAXTEK RQCM system. The mass loading of Ni-S during electrodeposition was measured on a NU-1000-covered (by the recently described Electrophoretic deposition), tin-oxide-coated gold QCM crystal $(6 \mathrm{MHz})$.

The Faradiac efficiency for $\mathrm{H}_{2}$ was determined by galvanostatic electrolysis at $2 \mathrm{~mA} \mathrm{~cm}^{-2}$ for $3 \mathrm{~h}$. The experiment was carried out in a 60 -ml Gamry 5-neck cell equipped with 3 Ace-Thred ports to hold each electrode and two joints capable of being sealed with septa for gas sparging. This setup included the NU-1000_Ni-S as the working electrode $\left(c a .0 .5 \mathrm{~cm}^{2}\right.$ surface area), a Pt wire counter electrode (flame annealed with a butane torch before use and separated from the bulk solution by fine glass frit) and a $\mathrm{Ag} / \mathrm{AgCl}$ reference electrode (leakless assembly, eDAQ). Outside of the electrolyte solution, a bare portion of the FTO working electrode was attached to a $\mathrm{Cu}$ wire by a minimal amount of non-conductive, chemically resistant epoxy, to attach the FTO glass to the potentiostat leads. A BASi Epsilon potentiostat was used to apply constant current and record potential. The electrolysis experiment was carried out in $30 \mathrm{ml}$ of total electrolyte solution $(0.1 \mathrm{M}$ $\mathrm{HCl}$ in water). The solution was constantly stirred throughout the experiment. Gas analysis was performed using $1 \mathrm{ml}$ sample injections on a Hewlett-Packard 7890A Series gas chromatograph with two molsieve columns $(30 \mathrm{~m} \times 0.53 \mathrm{~mm}$

ID $\times 25 \mu \mathrm{m}$ film). Gas chromatography calibration curve were made by sampling known volumes of $\mathrm{H}_{2}$ gas.

Upper limit TON and TOF were estimated in the following manner: we assume that only the portion of the metal sulfide that is in contact with the solution is catalytic (that is, the electrolyte does not permeate and swell the metal sulfide).
In addition, we assume that an adjacent pair of nickel-coordinated sulfide ions in the $\mathrm{Ni}_{3} \mathrm{~S}_{2}$ catalyst constitutes one HER catalytic site. Using the reported sulfur to sulfur distance in $\mathrm{Ni}_{3} \mathrm{~S}_{2}(3.5 \AA)$ as well as the ionic radius of $\mathrm{S}(1.7 \AA)$, a rough estimate of catalytic site area in $\mathrm{cm}^{2}$ was made (assuming a rectangular shaped site):

Catalytic site length $\left(3.5 \times 10^{-8}+\left(1.7 \times 10^{-8} \times 2\right)\right) \times$ catalytic site width $\left(1.7 \times 10^{-8} \times 2\right)=$ catalytic site area $\left(2.34 \times 10^{-15} \mathrm{~cm}^{2}\right)$. The number of catalytic sites per $1 \mathrm{~cm}^{2}$ is $4.26 \times 10^{+14}$; hence, there are $7.07 \times 10^{-10}$ moles of catalytic sites per $1 \mathrm{~cm}^{2}$. The number of moles of produced $\mathrm{H}_{2}$ during $8,000 \mathrm{~s}$ of electrolysis (taking into account $93 \% \mathrm{H}_{2}$ Faradaic efficiency) was calculated to be $1.94 \times 10^{-4}$

As a result, upper limit TON $=$ moles of $\mathrm{H}_{2}$ /moles of catalytic sites $(273,000)$.

Upper limit TOF $=$ upper limit TON/time of electrolysis $\left(34 \mathrm{~s}^{-1}\right)$.

TON and TOF values based on the total amount of Ni-S in the film

$\left(28 \mu \mathrm{g} \mathrm{cm}^{-2}, 1.16 \times 10^{-7}\right.$ moles) are 1,668 and 0.208 , respectively.

Impedance spectroscopy measurements under HER working conditions were made using an $\mathrm{AC}$ voltage of $20 \mathrm{mV}$, with a frequency range of $500 \mathrm{kHz}-50 \mathrm{mHz}$

Growth of NU-1000 thin films. The growth of NU-1000 films on glass-supported, transparent and electronically conductive, FTO electrodes ('FTO_NU-1000') was done using a slightly modified version of a previously reported solvothermal route $^{26}$. The FTO glass substrate $\left(15 \Omega^{-2}\right.$, Hartford Glass), with a size of $2.5 \times 1.25 \mathrm{~cm}$, was washed in soapy water, ethanol and acetone by sonication for 15 min sequentially. Thereafter, the substrate was dried and soaked in a solution of $0.5 \mathrm{mM} \mathrm{H}_{4}$ TBAPy in DMF at room temperature for $12 \mathrm{~h}$. The detailed synthesis of the $\mathrm{H}_{4}$ TBAPy has been reported in our previous work ${ }^{1}$. The substrate was then cleaned with DMF and dried. Benzoic acid $(2.7 \mathrm{~g})$ and $105 \mathrm{mg}$ of zirconyl chloride octahydrate were added into $8 \mathrm{ml}$ of DMF and ultrasonically dissolved in a $20-\mathrm{ml}$ screw-thread sample vial (Cole-Parmer, $28 \mathrm{~mm} \times 57 \mathrm{~mm}$ ), equipped with a urea cap and polytetrafluoroethylene (PTFE) foam-backed liner. Thereafter, the solution was placed into an oven at $80^{\circ} \mathrm{C}$ for $2 \mathrm{~h}$. After cooling down the solution to room temperature, $40 \mathrm{mg}$ of $\mathrm{H}_{4} \mathrm{TBAPy}$ was added into this solution and the mixture was sonicated for $20 \mathrm{~min}$. The as-prepared FTO substrate was then placed into the solution, with the conducting side facing down to the bottom. Subsequently, the vial was placed on the bottom of a gravity convection oven (VWR symphony) with the temperature set at $90^{\circ} \mathrm{C}$; the bottom of the oven provided a temperature gradient inside the vial, which is required for the growth of the MOF thin film. The vial was taken out from the oven after $13 \mathrm{~h}$ of reaction and the FTO substrate was taken out from the vial. After removing all the precipitations on the back side of the substrate, the substrate was washed with DMF; a uniform pale yellow MOF thin film could be observed on the front side of the FTO substrate. Benzoates (modulator) coordinated in the obtained MOF thin film was then removed by the following activation process: $0.5 \mathrm{ml}$ of $8 \mathrm{M}$ hydrochloric acid aqueous solution was mixed with $13 \mathrm{ml}$ of DMF; $0.05 \mathrm{ml}$ of the obtained solution was then mixed with $49.95 \mathrm{ml}$ of DMF to form the diluted acidic solution. The MOF thin film was then soaked in the diluted acidic solution in a $100^{\circ} \mathrm{C}$ oven for 4 days. Then, the film was washed with acetone for several times and soaked in acetone for 1 day. After drying the film in air, the FTO_NU-1000 thin film was then obtained. In a few cases, the $\mathrm{HCl}$ treatment was omitted and the benzoate-coordinating version of the material was used instead.

Electrodeposition of Ni-S. Potentiostatic electrodeposition of Ni-S on FTO_NU1000 ('NU-1000_Ni-S') and bare FTO ('FTO_Ni-S') films was conducted according to a slightly modified version of a previously published procedure ${ }^{23}$. Briefly, an aqueous solution containing $10 \mathrm{mM} \mathrm{NiCl}_{2}$ and $0.5 \mathrm{M}$ thiourea as nickel and sulfur sources, respectively, was used as the deposition bath. The electrodeposition was carried out in a standard three-electrode setup containing either FTO NU-1000 or bare FTO as a working electrode, $\mathrm{Ag} / \mathrm{AgCl}$ as a reference electrode and FTO as a counter electrode. To determine the potential at which the deposition should be done, CV measurements of the deposition solution with and without a sulfur source were recorded (see Supplementary Fig. 1). It is clear that on addition of the sulfur source, the reduction peak for $\mathrm{Ni}^{2}+/ \mathrm{Ni}^{0}$ ( $\mathrm{Ni}$ deposition) is shifted to more anodic potentials, from -1.35 to $-1.1 \mathrm{~V}$ versus $\mathrm{Ag} / \mathrm{AgCl}$, where the shift is a result of the reaction between the deposited $\mathrm{Ni}$ and thiourea (sulfur source) to generate $\mathrm{Ni}-\mathrm{S}^{23}$. As a consequence, all subsequent $\mathrm{Ni}-\mathrm{S}$ electrodepositions were carried out at a fixed potential of $-1.1 \mathrm{~V}$ versus $\mathrm{Ag} / \mathrm{AgCl}$.

Proton conductivity measurements. To measure the MOF's proton conductivity, disk pellets of NU-1000 (only) or benzoate modified NU-1000 were prepared, with dimensions of $7 \mathrm{~mm}$ diameter and $3 \mathrm{~mm}$ thickness. Each side of the pellet was coated with conductive silver epoxy, which was used to anchor a pair of tin-coated copper wires as electrical contacts. Then, the pellet was placed in an oven set to $60^{\circ} \mathrm{C}$ for half an hour, to cure the epoxy. The pellet was exposed to $\mathrm{H}_{2} \mathrm{O}$ vapour at ambient temperature and its impedance spectroscopy response based on an AC signal of $20 \mathrm{mV}$ was recorded over the frequency range of $500 \mathrm{kHz}$ to $0.5 \mathrm{~Hz}$.

\section{References}

1. Meyer, T. J. Chemical approaches to artificial photosynthesis. Accounts. Chem Res. 22, 163-170 (1989).

2. Lewis, N. S. \& Nocera, D. G. Powering the planet: chemical challenges in solar energy utilization. Proc. Natl Acad. Sci. USA 103, 15729-15735 (2006). 
3. Turner, J. A. Sustainable hydrogen production. Science 305, 972-974 (2004).

4. Gray, H. B. Powering the planet with solar fuel. Nat. Chem. 1, 7-7 (2009).

5. Walter, M. G. et al. Solar water splitting cells. Chem. Rev. 110, 6446-6473 (2010).

6. Merki, D. \& Hu, X. L. Recent developments of molybdenum and tungsten sulfides as hydrogen evolution catalysts. Energ. Environ. Sci. 4, 3878-3888 (2011).

7. Brown, D. E., Mahmood, M. N., Turner, A. K., Hall, S. M. \& Fogarty, P. O. Low overvoltage electrocatalysts for hydrogen evolving electrodes. Int. J. Hydrogen Energ. 7, 405-410 (1982).

8. Vrubel, H. \& Hu, X. L. Molybdenum boride and carbide catalyze hydrogen evolution in both acidic and basic solutions. Angew. Chem. Int. Ed. 51, 12703-12706 (2012).

9. Popczun, E. J. et al. Nanostructured nickel phosphide as an electrocatalyst for the hydrogen evolution reaction. J. Am. Chem. Soc. 135, 9267-9270 (2013).

10. Feng, L. G., Vrubel, H., Bensimon, M. \& Hu, X. L. Easily-prepared dinickel phosphide $\left(\mathrm{Ni}_{2} \mathrm{P}\right)$ nanoparticles as an efficient and robust electrocatalyst for hydrogen evolution. Phys. Chem. Chem. Phys. 16, 5917-5921 (2014).

11. Chen, W. F. et al. Hydrogen-evolution catalysts based on non-noble metal nickel-molybdenum nitride nanosheets. Angew. Chem. Int. Ed. 51, 6131-6135 (2012).

12. Jaramillo, T. F. et al. Identification of active edge sites for electrochemical H-2 evolution from $\mathrm{MoS}_{2}$ nanocatalysts. Science 317, 100-102 (2007).

13. Kong, D. S., Cha, J. J., Wang, H. T., Lee, H. R. \& Cui, Y. First-row transition metal dichalcogenide catalysts for hydrogen evolution reaction. Energ. Environ. Sci. 6, 3553-3558 (2013).

14. Kibsgaard, J., Chen, Z. B., Reinecke, B. N. \& Jaramillo, T. F. Engineering the surface structure of $\mathrm{MoS}_{2}$ to preferentially expose active edge sites for electrocatalysis. Nat. Mater. 11, 963-969 (2012).

15. Tran, P. D. et al. Novel cobalt/nickel-tungsten-sulfide catalysts for electrocatalytic hydrogen generation from water. Energ. Environ. Sci. 6, 2452-2459 (2013).

16. Voiry, D. et al. Enhanced catalytic activity in strained chemically exfoliated $\mathrm{WS}_{2}$ nanosheets for hydrogen evolution. Nat. Mater. 12, 850-855 (2013).

17. Li, Y. G. et al. $\mathrm{MoS}_{2}$ nanoparticles grown on graphene: an advanced catalyst for the hydrogen evolution reaction. J. Am. Chem. Soc. 133, 7296-7299 (2011).

18. Hinnemann, B. et al. Biornimetic hydrogen evolution: $\mathrm{MoS}_{2}$ nanoparticles as catalyst for hydrogen evolution. J. Am. Chem. Soc. 127, 5308-5309 (2005).

19. Layfield, J. P. \& Hammes-Schiffer, S. Hydrogen tunneling in enzymes and biomimetic models. Chem. Rev. 114, 3466-3494 (2014).

20. Cornish, A. J., Gartner, K., Yang, H., Peters, J. W. \& Hegg, E. L. Mechanism of proton transfer in $[\mathrm{FeFe}]$-hydrogenase from Clostridium pasteurianum. J. Biol. Chem. 286, 38341-38347 (2011).

21. Thorp, H. H., Sarneski, J. E., Brudvig, G. W. \& Crabtree, R. H. Proton-coupled electron-transfer in $[(\mathrm{Bpy}) 2 \mathrm{mn}(\mathrm{O}) 2 \mathrm{mn}(\mathrm{Bpy}) 2]^{3+}$. J. Am. Chem. Soc. 111, 9249-9250 (1989).

22. Bollinger, M. V. et al. One-dimensional metallic edge states in $\mathrm{MoS}_{2}$. Phys. Rev. Lett. 87, 196803-196804 (2001).

23. Sun, H. C. et al. Dye-sensitized solar cells with NiS counter electrodes electrodeposited by a potential reversal technique. Energ. Environ. Sci. 4, 2630-2637 (2011).

24. Jiang, N. et al. Electrodeposited nickel-sulfide films as competent hydrogen evolution catalysts in neutral water. J. Mater. Chem. A 2, 19407-19414 (2014).

25. Mondloch, J. E. et al. Vapor-phase metalation by atomic layer deposition in a metal-organic framework. J. Am. Chem. Soc. 135, 10294-10297 (2013).

26. Kung, C. W. et al. Metal-organic framework thin films composed of freestanding acicular nanorods exhibiting reversible electrochromism. Chem. Mater. 25, 5012-5017 (2013).

27. Katz, M. J. et al. A facile synthesis of UiO-66, UiO-67 and their derivatives. Chem. Commun. 49, 9449-9451 (2013).

28. Katz, M. J. et al. Simple and compelling biomimetic metal-organic framework catalyst for the degradation of nerve agent simulants. Angew. Chem. Int. Ed. 53, 497-501 (2014).

29. DeCoste, J. B. et al. Stability and degradation mechanisms of metal-organic frameworks containing the $\mathrm{Zr6O} 4(\mathrm{OH})(4)$ secondary building unit. J. Mater. Chem. A 1, 5642-5650 (2013).

30. Furukawa, H. et al. Water adsorption in porous metal-organic frameworks and related materials. J. Am. Chem. Soc. 136, 4369-4381 (2014).

31. Morris, W. et al. Synthesis, structure, and metalation of two new highly porous zirconium metal-organic frameworks. Inorg. Chem. 51, 6443-6445 (2012).

32. Wu, H., Yildirim, T. \& Zhou, W. Exceptional mechanical stability of highly porous zirconium metal-organic framework UiO-66 and its important implications. J. Phys. Chem. Lett. 4, 925-930 (2013).

33. Vermoortele, F., Ameloot, R., Vimont, A., Serre, C. \& De Vos, D. An aminomodified $\mathrm{Zr}$-terephthalate metal-organic framework as an acid-base catalyst for cross-aldol condensation. Chem. Commun. 47, 1521-1523 (2011).
34. Mondloch, J. E. et al. Are $\mathrm{Zr}_{6}$-based MOFs water stable? Linker hydrolysis vs. capillary-force-driven channel collapse. Chem. Commun. 50, 8944-8946 (2014).

35. Silva, C. G., Luz, I., Xamena, F. X. L. I., Corma, A. \& Garcia, H. Water stable Zrbenzenedicarboxylate metal-organic frameworks as photocatalysts for hydrogen generation. Chem. Eur. J. 16, 11133-11138 (2010).

36. He, J. et al. Significantly enhanced photocatalytic hydrogen evolution under visible light over CdS embedded on metal-organic frameworks. Chem. Commun. 49, 6761-6763 (2013).

37. Wang, J. L., Wang, C. \& Lin, W. B. Metal-organic frameworks for light harvesting and photocatalysis. ACS Catal. 2, 2630-2640 (2012).

38. Kataoka, Y. et al. Photocatalytic hydrogen production from water using porous material [Ru-2(p-BDC)(2)](n). Energ. Environ. Sci. 2, 397-400 (2009).

39. Wang, C., deKrafft, K. E. \& Lin, W. B. Pt nanoparticles@photoactive metalorganic frameworks: efficient hydrogen evolution via synergistic photoexcitation and electron injection. J. Am. Chem. Soc. 134, 7211-7214 (2012).

40. deKrafft, K. E., Wang, C. \& Lin, W. B. Metal-organic framework templated synthesis of $\mathrm{Fe}_{2} \mathrm{O}_{3} / \mathrm{TiO}_{2}$ nanocomposite for hydrogen production. Adv. Mater 24, 2014-2018 (2012).

41. Planas, N. et al. Defining the proton topology of the Zr-6-based metal-organic framework NU-1000. J. Phys. Chem. Lett. 5, 3716-3723 (2014).

42. Nesbitt, H. W., Legrand, D. \& Bancroft, G. M. Interpretation of Ni2p XPS spectra of Ni conductors and Ni insulators. Phys. Chem. Miner. 27, 357-366 (2000).

43. Cheng, Z., Abernathy, H. \& Liu, M. L. Raman spectroscopy of nickel sulfide $\mathrm{Ni}_{3} \mathrm{~S}_{2}$. J. Phys. Chem. C. 111, 17997-18000 (2007).

44. de las Heras, C. \& Agullo-Rueda, F. Raman spectroscopy of $\mathrm{NiSe}_{2}$ and $\mathrm{NiS}_{2-\mathrm{x}} \mathrm{Se}_{\mathrm{x}}(0<\mathrm{x}<2)$ thin films. J. Phys. Condens. Mater. 12, 5317-5324 (2000).

45. Guillaume, F., Huang, S., Harris, K. D. M., Couzi, M. \& Talaga, D. Optical phonons in millerite (NiS) from single-crystal polarized Raman spectroscopy. J. Raman Spectrosc. 39, 1419-1422 (2008).

46. Sun, Y. J. et al. Electrodeposited cobalt-sulfide catalyst for electrochemical and photoelectrochemical hydrogen generation from water. J. Am. Chem. Soc. 135, 17699-17702 (2013).

47. Zeng, K. \& Zhang, D. K. Recent progress in alkaline water electrolysis for hydrogen production and applications. Prog. Energ. Combust. 36, 307-326 (2010).

48. Bockris, J. O. M. \& Potter, E. C. The mechanism of the cathodic hydrogen evolution reaction. J. Electrochem. Soc. 99, 169-186 (1952).

49. Vrubel, H., Moehl, T., Gratzel, M. \& Hu, X. L. Revealing and accelerating slow electron transport in amorphous molybdenum sulphide particles for hydrogen evolution reaction. Chem. Commun. 49, 8985-8987 (2013).

50. McCrory, C. C. L., Jung, S. H., Peters, J. C. \& Jaramillo, T. F. Benchmarking heterogeneous electrocatalysts for the oxygen evolution reaction. J. Am. Chem. Soc. 135, 16977-16987 (2013).

51. Deria, P., Bury, W., Hupp, J. T. \& Farha, O. K. Versatile functionalization of the NU-1000 platform by solvent-assisted ligand incorporation. Chem. Commun. 50, 1965-1968 (2014).

52. Deria, P. et al. Perfluoroalkane functionalization of NU-1000 via solventassisted ligand incorporation: synthesis and $\mathrm{CO}_{2}$ adsorption studies. J. Am. Chem. Soc. 135, 16801-16804 (2013).

53. Hod, I. et al. Bias-switchable permselectivity and redox catalytic activity of a ferrocene-functionalized, thin-film metal-organic framework compound. J. Phys. Chem. Lett. 6, 586-591 (2015).

54. Shigematsu, A., Yamada, T. \& Kitagawa, H. Wide control of proton conductivity in porous coordination polymers. J. Am. Chem. Soc. 133, 2034-2036 (2011).

55. Kundu, T., Sahoo, S. C. \& Banerjee, R. Alkali earth metal (Ca, Sr, Ba) based thermostable metal-organic frameworks (MOFs) for proton conduction. Chem Commun. 48, 4998-5000 (2012).

56. Horike, S., Umeyama, D. \& Kitagawa, S. Ion conductivity and transport by porous coordination polymers and metal-organic frameworks. Accounts. Chem. Res 46, 2376-2384 (2013).

57. Taylor, J. M., Dawson, K. W. \& Shimizu, G. K. H. A water-stable metal-organic framework with highly acidic pores for proton-conducting applications. J. Am. Chem. Soc. 135, 1193-1196 (2013).

58. Wiedner, E. S., Appel, A. M., DuBois, D. L. \& Bullock, R. M. Thermochemical and mechanistic studies of electrocatalytic hydrogen production by cobalt complexes containing pendant amines. Inorg. Chem. 52, 14391-14403 (2013).

59. Wiese, S. et al. Hydrogen production using nickel electrocatalysts with pendant amines: ligand effects on rates and overpotentials. ACS Catal. 3, 2527-2535 (2013).

60. Liu, T. B., DuBois, D. L. \& Bullock, R. M. An iron complex with pendent amines as a molecular electrocatalyst for oxidation of hydrogen. Nat. Chem. 5, 228-233 (2013). 
61. Dutta, A. et al. Minimal proton channel enables H-2 oxidation and production with a water-soluble nickel-based catalyst. J. Am. Chem. Soc. 135, 18490-18496 (2013).

62. Chng, L. L., Chang, C. J. \& Nocera, D. G. Catalytic O-O activation chemistry mediated by iron hangman porphyrins with a wide range of proton-donating abilities. Org. Lett. 5, 2421-2424 (2003).

63. Costentin, C., Drouet, S., Robert, M. \& Saveant, J. M. A local proton source enhances $\mathrm{CO}_{2}$ electroreduction to $\mathrm{CO}$ by a molecular Fe catalyst. Science 338, 90-94 (2012).

\section{Acknowledgements}

We thank the following for postdoctoral or graduate fellowship support: the Fulbright Commission (I.H.), the Graduate Students Study Abroad Program sponsored by National Science Council, Taiwan (C.W.K.), the National Defense Science and Engineering Graduate (NDSEG) Fellowship programme (M.S.) and a grant from the Air Force Office of Scientific Research, MURI programme (Award Number FA9550-10-1-0572) (M.D.S). J.T.H and O.K.F acknowledge that this work was supported as part of the ANSER Center, an Energy Frontier Research Center funded by the U.S. Department of Energy, Office of Science, Office of Basic Energy Sciences under Award Number DE-SC0001059.

\section{Author contributions}

J.T.H., O.K.F and I.H. conceived and designed the experiments. P.D. and W.B. participated in the discussions and analysis of data. J.E.M. and A.W.P. performed the ICP measurements. C.W.K. assisted in SEM and EDS analysis. M.S. performed the XPS measurements, while M.D.S. and C.P.K. conducted and analysed the $\mathrm{H}_{2}$ Faradaic efficiency measurements. I.H., J.T.H and O.K.F wrote the manuscript.

\section{Additional information}

Supplementary Information accompanies this paper at http://www.nature.com/ naturecommunications

Competing financial interests: The authors declare no competing financial interest.

Reprints and permission information is available online at http://npg.nature.com/ reprintsandpermissions/

How to cite this article: Hod, I. et al. A porous, proton-relaying, metal-organic framework material that accelerates electrochemical hydrogen evolution. Nat. Commun. 6:8304 doi: $10.1038 /$ ncomms9304 (2015).

(c) (i)

This work is licensed under a Creative Commons Attribution 4.0 International License. The images or other third party material in this article are included in the article's Creative Commons license, unless indicated otherwise in the credit line; if the material is not included under the Creative Commons license, users will need to obtain permission from the license holder to reproduce the material. To view a copy of this license, visit http://creativecommons.org/licenses/by/4.0/ 